\title{
Activating Room-Temperature Phosphorescence of Organic Luminophores via External Heavy-Atom Effect and Rigidity of Ionic Polymer Matrix
}

Zi-Ang Yan, Xiaohan Lin, Siyu Sun, Xiang Ma*, He Tian

Key Laboratory for Advanced Materials and Feringa Nobel Prize Scientist Joint Research Center, Frontiers Science Center for Materiobiology and Dynamic Chemistry, Institute of Fine Chemicals, School of Chemistry and Molecular Engineering, East China University of Science \& Technology; No. 130 Meilong Road, Shanghai 200237, China

*Corresponding author. Email: maxiang@ecust.edu.cn

\begin{abstract}
Pure organic room-temperature phosphorescence (RTP) materials have attracted wide attention for their easy preparation, low toxicity and applications in professional fields such as bioimaging and anti-counterfeiting. Developing phosphorescent systems with more universality and less difficulty in synthesis has long been the pursuit of materials scientists. By employing polymeric quaternary ammonium salt with an ionic bonding matrix and heavy atoms, commercial fluorescent dyes are directly endowed with phosphorescence emission. In a single amorphous polymer, the external heavy-atom effect generates excited triplet states, which are further stabilized by the rigid polymer matrix. This study proposed a new general strategy to design and develop pure organic RTP materials starting from the vast library of organic dyes without complicated chemical synthesis.
\end{abstract}




\section{Main Text:}

Photoluminescent materials, including fluorescent and phosphorescent materials, have become a research spotlight since their discovery $(1-5)$. Compared with fluorescent materials, materials with room-temperature phosphorescence (RTP) have received special attention for their larger Stokes shift and longer lifetime (6-13). These advantages promote their applications in the fields such as molecular switches (14-18), organic light-emitting diodes (OLEDs) (19-23), anticounterfeiting (24) and bioimaging (25). As pure organic molecules typically emit phosphorescence only at low temperature (e.g., $77 \mathrm{~K})$ and under inert conditions $(26,27)$, traditional phosphorescent materials focus on inorganic and organometallic systems $(28,29)$, which normally rely on noble metals like iridium and platinum to promote the intersystem crossing (ISC) process. Therefore, the development of metal-free organic RTP materials is essential in order to avoid using these expensive and toxic heavy metals $(30,31)$.

It is known that the ISC process from a singlet state to a triplet state is a spin-forbidden process, and that the long-lived triplet excitons of organic molecules are vulnerable to free motions and triplet quenchers like oxygen, thus resulting in nonradiative deactivation. Recently, crystallizationinduced phosphorescence has become effective in constructing RTP materials (32-36), while this strategy remains restricted by strict growing conditions of crystals and low processability.

Several metal-free amorphous phosphorescent systems have been designed to overcome these disadvantages. Heavy atoms like bromine and iodine are modified onto luminophores to promote ISC with enhanced spin-orbit coupling. Methods like copolymerization and host-guest interaction are commonly used to inhibit nonradiative decay of phosphors (37-45). However, these methods generally involve complex synthesis techniques and sophisticated molecular designs to modify molecules with heavy atoms, vinyl groups and other necessary functional groups. These difficulties prevent scientists and engineers from utilizing the vast library of fluorescent dyes to develop phosphorescent materials. Consequently, it is necessary to design a universal method to 
conveniently endow traditional dyes with phosphorescence emission. Yet, the external heavy-atom effect and the rigidity of ionic polymers are phenomena known $(46,47)$ but seldom used to jointly active phosphorescence.

Herein, a general strategy was proposed for pure organic, amorphous RTP materials. Starting from commercially available polymeric quaternary ammonium salt, a phosphorescent system was designed by simply doping existing fluorescent dyes into a dual-functional polymer matrix (PAB, Fig. 1a), making them directly endowed with phosphorescence emission. The bromine ions in PAB enhance the spin-orbit coupling of these common fluorescent molecules via external heavy-atom effect, facilitating the ISC process from excited singlet to triplet states. These anions not merely act as heavy atoms, but also form a rigid ionic bonding network with the quaternary ammonium cations on the polymer backbone. With the aid of the rigid environment provided by $\mathrm{PAB}$, the quenchers are shielded and free motions of organic dyes are inhibited, so as to stabilize the triplet excitons and further suppress non-radiative transitions. Simply put, the generation and stabilization of excited triplet states is achieved with a single polymer matrix, which provides a convenient approach to activate RTP of organic molecules.

Several commercially available, frequently-used fluorescent dyes with literally no modifications were chosen as dopant. 1,8-naphthalimide (NpA), (S)-1,1'-bi-2-naphthol (S-BN), 6,7dihydroxycoumarin $(\mathrm{HCM})$, quinine sulfate $(\mathrm{QN})$, rhodamine $\mathrm{B}(\mathrm{RhB})$ and 3,6-diaminoacridine hydrochloride (PF) were respectively doped into poly-diallyldimethylammonium bromide (PAB) to form a film (Fig. 1b, 1c, for details see Supporting Information). For comparison purposes, polydiallyldimethylammonium chloride (PAC, Fig. 1a) was also used as a rigid matrix with similar ionic bonding network but without heavy atoms. The resulting films were represented by dye@matrix, where dye = NpA, S-BN, HCM, QN, RhB or PF; matrix = PAB or PAC.

As expected, all dye@PAB films exhibited obvious RTP emission. Due to distinct intrinsic properties of fluorescent dopants, doped systems showed different RTP emissions, respectively 
(Fig. 2). In comparison, all dye@PAC films still emitted fluorescence but extremely weak phosphorescence under the same testing conditions as a result of insufficient intersystem crossing of the dyes without the aid of heavy atoms, which in turn indicates the effectiveness of bromine ions in $\mathrm{PAB}$.

The brominated analogues of NpA have been extensively used and reported in constructing RTP materials for their large Stokes shift $(41,48,49)$. As shown in Fig. 2a, similar phosphorescence spectrum of NpA was obtained when doped in PAB film, suggesting that the external heavy atoms acted just like the covalently linked ones and that the nonradiative relaxation of $\mathrm{NpA}$ was effectively suppressed by the ionic bonding network. While the delayed spectrum of NpA@PAB contrasted sharply to that of NpA@PAC, phosphorescence lifetimes can serve as the most convincing evidence of external heavy-atom effect, which accelerates the transition both from $\mathrm{S}_{1}$ to $T_{1}$ and from $T_{1}$ to $S_{0}$, decreasing the phosphorescence lifetime. Although the RTP signal of NpA@PAC was very weak, fortunately it is still detectable. Phosphorescence lifetime measurement was carried out at the same excitation and emission wavelength for NpA@PAB and NpA@PAC. Results show that NpA@PAC had a RTP lifetime of 39.142 ms, a clear indication of slow $\mathrm{T}_{1} \rightarrow \mathrm{S}_{0}$ transition without heavy atoms. In contrast, the lifetime of $\mathrm{NpA} @ \mathrm{PAB}$ was $3.721 \mathrm{~ms}$, which is an order of magnitude decrease. Upon removal of $365 \mathrm{~nm} \mathrm{UV}$ irradiation, very weak afterglow was observed from NpA@PAC, but no afterglow can be seen from NpA@PAB due to the shortness of its lifetime.

Widely used in the fields like photophysics and photochemistry, S-BN, HCM and QN are all effectively endowed with RTP characters when doped in PAB films. Similar to the case of NpA, these dyes show very weak phosphorescence in $0.1 \mathrm{~ms}$ delayed spectra but exhibit longer lifetimes (for comparison, see Table S1) when doped in PAC. Since the long lifetimes of S-BN and HCM in $\mathrm{PAB}$ remain observable by naked eyes, they show significantly brighter but short-lived afterglow after removal of UV light, compared with those doped in PAC (Fig. 2b-2d). 
For dye@PAB, noticeable differences between the emission maxima of prompt and delayed spectra mentioned above confirms the existence of RTP, instead of delayed fluorescence. For some organic luminophores, however, the generation of excited triplet states can also lead to thermally activated delayed fluorescence (TADF) apart from phosphorescence. RhB is such a molecule with a small HOMO-LUMO overlap integral. The delayed spectrum of RhB@PAB shows an emission maximum at $605 \mathrm{~nm}$ and a small peak at $c a .650 \mathrm{~nm}$, overlapping well with its prompt spectrum. This can be attributed to TADF. The delayed spectrum also exhibits a new peak considered to be phosphorescence at $c a .700 \mathrm{~nm}$. To confirm the existence of TADF, temperature-dependent delayed spectra was acquired by putting RhB@PAB into a temperature-controlled quartz holder, whose temperature changes from $20^{\circ} \mathrm{C}$ to $70^{\circ} \mathrm{C}$. Fig. 3a shows that the intensity of TADF first increased with elevating temperature due to the thermally activated nature of TADF. Since the faster consumption of excited triplet states leads to shorter emission lifetime (see Fig. 3d), the intensity after $0.1 \mathrm{~ms}$ delay time with $2.0 \mathrm{~ms}$ gate time eventually decreased, despite stronger initial emission. Unlike the above cases, the phosphorescence and delayed fluorescence in RhB@PAC was too weak to be detected.

According to Fig. $2 \mathrm{f}$ and $3 \mathrm{~b}, \mathrm{PF}$ is a molecule that intrinsically possess a degree of ISC and reverse ISC since long-lived TADF can be observed in PF@PAC, whose intensity increases with rising temperature. However, to activate phosphorescence, external heavy atoms are still needed. Owing to enhanced $\mathrm{T}_{1} \rightarrow \mathrm{S}_{0}$ transition induced by PAB, PF@PAB exhibited an obvious RTP emission at $581 \mathrm{~nm}$ besides delayed fluorescence, whose temperature-dependent behavior was in consistent with RhB@PAB, as revealed in Fig. 3c and 3f. The RhB@PAB and PF@PAB show that this doping system has extensive applications, including other phenomena involving triplet states like TADF.

So far, phosphorescence properties of dye@PAB materials have been investigated with both intuitive and solid evidences, verifying the universality of this novel and convenient design strategy 
proposed in this study. The phosphorescence and fluorescence properties of these materials are summarized in Table 1. The absorption also falls within the range of typical absorption spectra of the dopants, respectively (Fig. S3). It is safe to conclude that this strategy is applicable to a wide range of, though may not all of, organic luminophores, whose luminescence efficiencies vary in accordance with their intrinsic properties.

As can be seen in Fig. S4, X-ray powder diffraction (XRPD) analysis of PAC, PAB and all doped materials were carried out. XRPD diagrams show that there was no obvious crystal structure in the two polymer matrices and that the doping of dyes did not significantly affect this amorphous state, indicating that dye@PAB phosphorescent materials do not require strict growing conditions as crystals. The amorphous state also ensures better processability, which is an important character for phosphorescent materials. Similar amorphous states of PAC and PAB also provide comparability between their spectra. Like all other water-soluble matrices, the RTP of dye@PAB can be quenched by water and its dissolved oxygen, as exemplified in Fig. S5. Additionally, blank experiment was conducted by doping non-conjugated glucose (Glu) into PAB. Glu@PAB shows no RTP emission within the range of excitation wavelengths used for dye@PAB materials (Fig. S6).

In conclusion, the two obstacles in activating room-temperature phosphorescence, namely the generation and stabilization of excited triplet states, can be overcome by using a single ionic polymer matrix. The external heavy-atom effect promotes the intersystem crossing process of dopants, whilst the rigid ionic bonding matrix reduces the nonradiative deactivation. Furthermore, the absence of unsaturated groups in PAB minimizes possible quenching effects. All commercial fluorescent dyes used in this study were effectively endowed with phosphorescence characters without complicated modifications. Therefore, this work provides material scientists not only a practical and economical strategy to develop RTP materials, but also a powerful tool for studying the triplet states of organic compounds. 


\section{References and Notes}

1. H. Noda, X.-K. Chen, H. Nakanotani, T. Hosokai, M. Miyajima, N. Notsuka, Y. Kashima, J.-L. Brédas, C. Adachi, Critical role of intermediate electronic states for spin-flip processes in chargetransfer-type organic molecules with multiple donors and acceptors. Nat. Mater. 18, 1084-1090 (2019).

2. W. S. Andrews, Notes on fluorescence and phosphorescence. Science 19, 435-436 (1904).

3. Z. Yan, L. Zou, X. Ma, Recent advances in pure organic luminescent supramolecular materials. Chin. J. Org. Chem. 40, 1814-1822 (2020).

4. Y. Wang, X. Bin, X. Chen, S. Zheng, Y. Zhang. W. Z. Yuan, Emission and emissive mechanism of nonaromatic oxygen clusters. Macromol. Rapid Commun. 39, 1800528 (2018).

5. H. Li, H. Li, W. Wang, Y. Tao, S. Wang, Q. Yang, Y. Jiang, C. Zheng, W. Huang, R. Chen, Stimuli-responsive circularly polarized organic ultralong room temperature phosphorescence. Angew. Chem. Int. Ed. 59, 4756-4762 (2020).

6. B. Chen, W. Huang, H. Su, H. Miao, X. Zhang, G. Zhang, Unexpected chromophore-solvent reaction leads to bicomponent aggregation-induced phosphorescence. Angew. Chem. Int. Ed. 59, 10023-10026 (2020).

7. M. Calvin, G. D. Dorough, The phosphorescence of chlorophyll and some chlorin derivatives. Science 105, 433-434 (1947).

8. L. Gu, H. Wu, H. Ma, W. Ye, W. Jia, H. Wang, H. Chen, N. Zhang, D. Wang, C. Qian, Z. An, W. Huang, Y. Zhao, Color-tunable ultralong organic room temperature phosphorescence from a multicomponent copolymer. Nat. Commun. 11, 944 (2020).

9. W. Huang, B. Chen, G. Zhang, Persistent room-temperature radicals from anionic naphthalimides: spin paring and supramolecular chemistry. Chem. Eur. J. 25, 12497 (2019). 
10. F. Li, S. Guo, Y. Qin, Y. Shi, M. Han, Z. An, S. Liu, Q. Zhao, W. Huang, Achieving dual persistent room-temperature phosphorescence from polycyclic luminophores via inter/intramolecular charge transfer. Adv. Opt. Mater. 7, 1900511 (2019).

11. Q. Liao, Q. Gao, J. Wang, Y. Gong, Q. Peng, Y. Tian, Y. Fan, H. Guo, D. Ding, Q. Li, Z. Li, 9,9-Dimethylxanthene derivatives with room-temperature phosphorescence: substituent effects and emissive properties. Angew. Chem. Int. Ed. 59, 9946-9951 (2020).

12. E. L. Nichols, Some recent advances in fluorescence and phosphorescence. Science 33, 696$700(1911)$

13. Z. Yang, Z. Mao, X. Zhang, D. Ou, Y. Mu, Y. Zhang, C. Zhao, S. Liu, Z. Chi, J. Xu, Y.-C. Wu, P.-Y. Liu, A. Lien, M. R. Bryce, Intermolecular electronic coupling of organic units for efficient persistent room-temperature phosphorescence. Angew. Chem. Int. Ed. 55, 2181-2185 (2016).

14. T. Wang, Z. Hu, X. Nie, L. Huang, M. Hui, X. Sun, G. Zhang, Thermochromic aggregationinduced dual phosphorescence via temperature-dependent $\mathrm{sp}^{3}$-linked donor-acceptor electronic coupling. Nat. Commun. 12, 1364 (2021).

15. H. Wu, W. Chi, G. Baryshnikov, B. Wu, Y. Gong, D. Zheng, X. Li, Y. Zhao, X. Liu, H. Ågren, L. Zhu, Crystal multi-conformational control through deformable carbon-sulfur bond for singlettriplet emissive tuning. Angew. Chem. Int. Ed. 58, 4328-4333 (2019).

16. C. Li, X. Tang, L. Zhang, C. Li, Z. Liu, Z. Bo, Y. Q. Dong, Y.-H. Tian, Y. Dong, B. Z. Tang, Reversible luminescence switching of an organic solid: controllable on-off persistent room temperature phosphorescence and stimulated multiple fluorescence conversion. Adv. Opt. Mater. 3, 1184-1190 (2015).

17. X. Lin, J. Wang, B. Ding, X. Ma, H. Tian, Tunable-emission amorphous room-temperature phosphorescent polymers based on thermoreversible dynamic covalent bonds. Angew. Chem. Int. Ed. 60, 3459-3463 (2021). 
18. C. Chen, R. Huang, A. S. Batsanov, P. Pander, Y.-T. Hsu, Z. Chi, F. B. Dias, M. R. Bryce, Intermolecular charge transfer controls switching between room temperature phosphorescence and thermally activated delayed fluorescence. Angew. Chem. Int. Ed. 57, 16407-16411 (2018).

19. B. S. B. Karunathilaka, U. Balijapalli, C. A. M. Senevirathne, S. Yoshida, Y. Esaki, K. Goushi, T. Matsushima, A. S. D. Sandanayaka, C. Adachi, Suppression of external quantum efficiency rolloff in organic light emitting diodes by scavenging triplet excitons. Nat. Commun. 11, 4926 (2020).

20. X. Li, S. Shen, C. Zhang, M. Liu, J. Lu, L. Zhu, Small-molecule based thermally activated delayed fluorescence materials with dual-emission characteristics. Sci. China Chem. 64, 534-546 (2021).

21. N. Notsuka, R. Kabe, K. Goushi, C. Adachi, Confinement of long-lived triplet excitons in organic semiconducting host-guest systems. Adv. Funct. Mater. 27, 1703902 (2017).

22. T. Wang, X. Su, X. Zhang, X. Nie, L. Huang, X. Zhang, X. Sun, Y. Luo, G. Zhang, Aggregation-induced dual-phosphorescence from organic molecules for nondoped light-emitting diodes. Adv. Mater. 31, 1904273 (2019).

23. K. Yamaguchi, T. Matsushima, A. S. D. Sandanayaka, Y. Homma, N. Uchida, C. Adachi, Enhanced operational durability of thermally activated delayed fluorescence-based organic lightemitting diodes with a triazine electron transporter. Chem. Eur. J. 26, 5598-5602 (2020).

24. Y. Su, S. Z. F. Phua, Y. Li, X. Zhou, D. Jana, G. Liu, W. Q. Lim, W. K. Ong, C. Yang, Y. Zhao, Ultralong room temperature phosphorescence from amorphous organic materials toward confidential information encryption and decryption. Sci. Adv. 4, eaas9732 (2018).

25. Q. Dang, Y. Jiang, J. Wang, J. Wang, Q. Zhang, M. Zhang, S. Luo, Y. Xie, K. Pu, Q. Li, Z. Li, Room-temperature phosphorescence resonance energy transfer for construction of near-infrared afterglow imaging agents. Adv. Mater. 32, 2006752 (2020). 
26. Y. Mu, B. Xu, Z. Yang, H. Wen, Z. Yang, S. K. B. Mane, J. Zhao, Y. Zhang, Z. Chi, B. Z. Tang, Reversible and continuous color-tunable persistent luminescence of metal-free organic materials by "self"-interface energy transfer. ACS Appl. Mater. Interfaces 12, 5073-5080 (2020).

27. X.-F. Wang, H. Xiao, P.-Z. Chen, Q.-Z. Yang, B. Chen, C.-H. Tung, Y.-Z. Chen, L.-Z. Wu, Pure organic room temperature phosphorescence from excited dimers in self-assembled nanoparticles under visible and near-infrared irradiation in water. J. Am. Chem. Soc. 141, 50455050 (2019).

28. X. Yang, D. Yan, Long-afterglow metal-organic frameworks: reversible guest-induced phosphorescence tunability. Chem. Sci. 7, 4519-4526 (2016).

29. X. Yang, X. Lin, Y. Zhao, Y. S. Zhao, D. Yan, Lanthanide metal-organic framework microrods: colored optical waveguides and chiral polarized emission. Angew. Chem. Int. Ed. 56, 7853-7857 (2017).

30. Y. Tian, X. Yang, Y. Gong, Y. Wang, M. Fang, J. Yang, Z. Tang, Z. Li, The initial attempt to reveal the emission processes of both mechanoluminescence and room temperature phosphorescence with the aid of circular dichroism in solid state. Sci. China Chem. 64, 445-451 (2021).

31. X.-F. Wang, W.-J. Guo, H. Xiao, Q. Z. Yang, B. Chen, Y.-Z. Chen, C.-H. Tung, L.-Z. Wu, Pure organic room temperature phosphorescence from unique micelle-assisted assembly of nanocrystals in water. Adv. Funct. Mater. 30, 1907282 (2020).

32. Z. Yang, C. Xu, W. Li, Z. Mao, X. Ge, Q. Huang, H. Deng, J. Zhao, F. L. Gu, Y. Zhang, Z. Chi, Boosting the quantum efficient of ultralong organic phosphorescence up to $52 \%$ via intramolecular halogen bonding. Angew. Chem. Int. Ed. 59, 17451-17455 (2020). 
33. Z. Yin, M. Gu, H. Ma, X. Jiang, J. Zhi, Y. Wang, H. Yang, W. Zhu, Z. An, Molecular engineering through control of structural deformation for highly efficient ultralong organic phosphorescence. Angew. Chem. Int. Ed. 60, 2058-2063 (2021).

34. X. Zhou, S. Xu, L. Liu, Y. Sun, J. Cheng, X. Duan, L. Zhou, H. Qu, 5,5-Dioxoyphenothiazinebased D-A-D type AIE molecules enabling persistent room temperature phosphorescence, white light emission and dual-mode mechanochromism. Dyes Pigm. 188, 109193 (2021).

35. Y. Gong, G. Chen, Q. Peng, W. Z. Yuan, Y. Xie, S. Li, Y. Zhang, B. Z. Tang, Achieving persistent room temperature phosphorescence and remarkable mechanochromism from pure organic luminogens. Adv. Mater. 27, 6195-6201 (2015).

36. Y. Gong, L. Zhao, Q. Peng, D. Fan, W. Z. Yuan, Y. Zhang, B. Z. Tang, Crystallization-induced dual emission from metal- and heavy atom-free aromatic acids and esters. Chem. Sci. 6, 4438-4444 (2015).

37. Z. Wang, T. Li, B. Ding, X. Ma, Achieving room temperature phosphorescence from organic small molecules on amino acid skeleton. Chin. Chem. Lett. 31, 2929-2932 (2020).

38. B. Wu, H. Wu, Y. Gong, A. Li, X. Jia, L. Zhu, A chiral single-component sol-gel platform with highly integrated optical properties. J. Mater. Chem. C 9, 4275-4280 (2021).

39. H. Wu, W. Chi, Z. Chen, G. Liu, L. Gu, A. K. Bindra, G. Yang, X. Liu, Y. Zhao, Achieving amorphous ultralong room temperature phosphorescence by coassembling planar small organic molecules with polyvinyl alcohol. Adv. Funct. Mater. 29, 1807243 (2019)

40. T. Zhang, X. Ma, H. Wu, L. Zhu, Y. Zhao, H. Tian, Molecular engineering for metal-free amorphous materials with room-temperature phosphorescence. Angew. Chem. Int. Ed. 59, 11206$11216(2020)$.

41. H. Chen, X. Yao, X. Ma, H. Tian, Amorphous, efficient, room-temperature phosphorescent metal-free polymers and their applications as encryption ink. Adv. Opt. Mater. 4, 1397-1401 (2016). 
42. M. S. Kwon, D. Lee, S. Seo, J. Jung, J. Kim, Tailoring intermolecular interactions for efficient room-temperature phosphorescence from purely organic materials in amorphous polymer matrices. Angew. Chem. Int. Ed. 53, 11177-11181 (2014).

43. M. S. Kwon, Y. Yu, C. Coburn, A. W. Phillips, K. Chung, A. Shanker, J. Jung, G. Kim, K. Pipe, S. R. Forrest, J. H. Youk, J. Gierschner, J. Kim, Suppressing molecular motions for enhanced room-temperature phosphorescence of metal-free organic materials. Nat. Commun. 6, 8947 (2015). 44. T. Ogoshi, H. Tsuchida, T. Kakuta, T.-a. Yamagishi, A. Taema, T. Ono, M. Sugimoto, M. Mizuno, Ultralong room-temperature phosphorescence from amorphous polymer poly(styrene sulfonic acid) in air in the dry solid state. Adv. Funct. Mater. 28, 1707369 (2018).

45. H. Chen, X. Ma, S. Wu, H. Tian, A rapid self-healing supramolecular polymer hydrogel with photostimulated room-temperature phosphorescence responsiveness. Angew. Chem. Int. Ed. 53, 14149-14152 (2014).

46. N. J. Turro, V. Ramamurthy, J. C. Scaiano, "Radiative Transitions between Electronic States" \& "Photophysical Radiationless Transitions" in Modern Molecular Photochemistry of Organic Molecules (University Science Books, Sausalito, California, 2010), pp. 240-243, 313.

47. S. Cai, H. Ma, H. Shi, H. Wang, X. Wang, L. Xiao, W. Ye, K. Huang, X. Cao, N. Gan, C. Ma, M. Gu, L. Song, H. Xu, Y. Tao, C. Zhang, W. Yao, Z. An, W. Huang, Enabling long-lived organic room temperature phosphorescence in polymers by subunit interlocking. Nat. Commun. 10, 4247 (2019).

48. D. Wang, Z. Yan, M. Shi, J. Dai, Q. Chai, H. Gui, Y. Zhang, X. Ma, Employing lactam copolymerization strategy to effectively achieve pure organic room-temperature phosphorescence in amorphous state. Adv. Opt. Mater. 7, 1901277 (2019). 
49. X. Ma, J. Wang, H. Tian, Assembling-induced emission: an efficient approach for amorphous metal-free organic emitting materials with room-temperature phosphorescence. Acc. Chem. Res. 52, 738-748 (2019).

\section{Acknowledgments:}

\section{Funding:}

National Natural Science Foundation of China grant 21788102, 21722603 and 21871083

Program of Shanghai Academic/Technology Research Leader grant 20XD1421300

'Shu Guang' project supported by Shanghai Municipal Education Commission and Shanghai Education Development Foundation grant 19SG26

The Innovation Program of Shanghai Municipal Education Commission grant 2017-01-07-00-

02-E00010

Fundamental Research Funds for the Central Universities

\section{Author contributions:}

Conceptualization: ZY, XM, HT

Investigation: ZY

Methodology: ZY

Formal Analysis: ZY

Funding acquisition: XM, HT

Project administration: XM, HT

Supervision: XM, HT

Writing - original draft: ZY

Writing - review \& editing: ZY, XL, XM, HT, SS 
Competing interests: Authors declare that they have no competing interests.

Data and materials availability: All data are available in the main text or the supplementary materials.

\section{Supplementary Materials}

General experimental procedures

Materials

Figs. S1 to S6

Table S1 


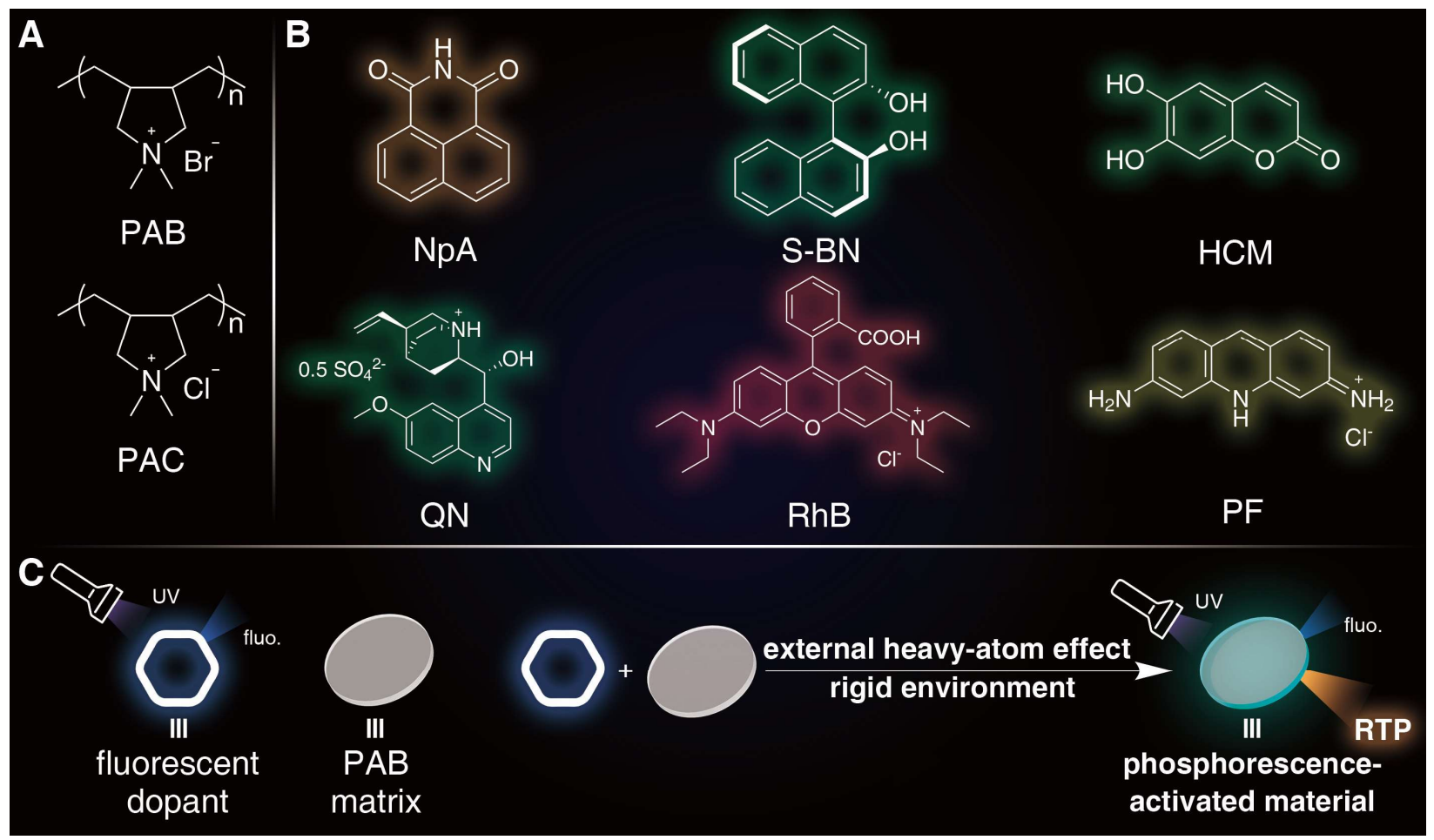

Fig. 1. (A) Structures of the polymers: PAB and PAC. (B) Structures of the dopants: NpA, S-BN, HCM, QN, RhB and PF. colors are applied according to the delayed spectra of dye@PAB. (C) Illustration of the general strategy.

A

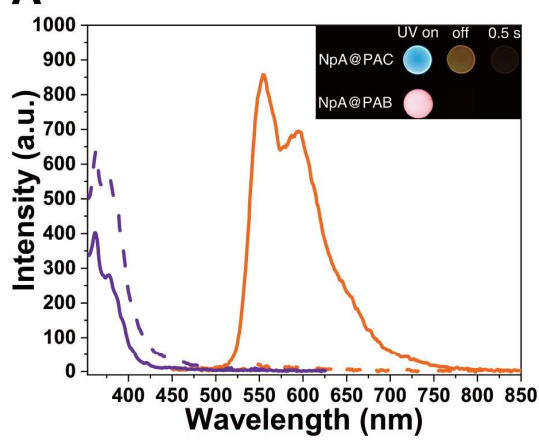

D

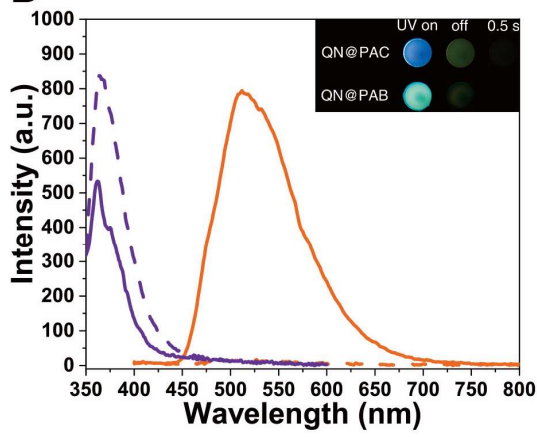

B

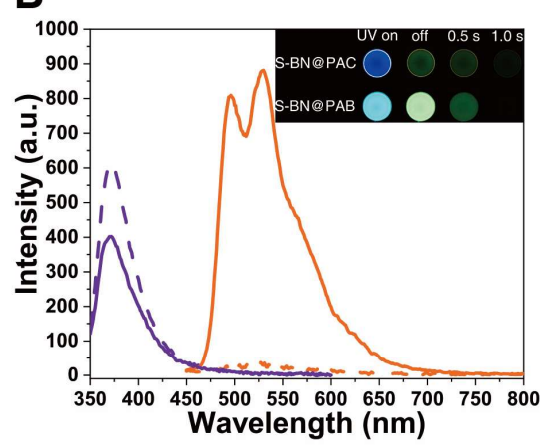

$E$

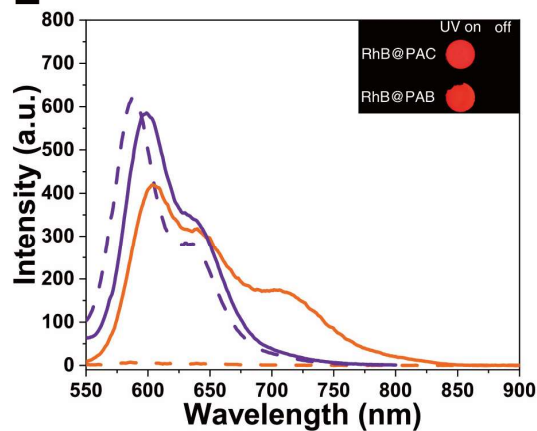

C

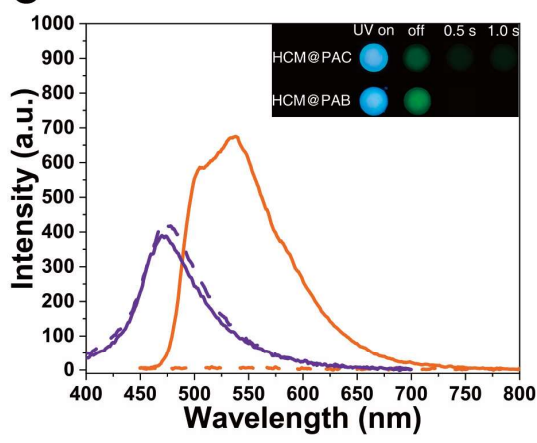

$\mathbf{F}$

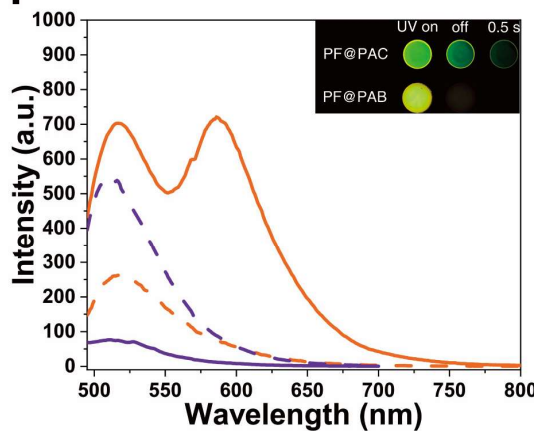


Fig. 2. Prompt (purple lines) and delayed (orange lines) spectra of dye@PAC (dashed line) and dye@PAB (solid line), where dye = (A) NpA; (B) S-BN; (C) HCM; (D) QN; (E) RhB; (F) PF. Smoothed, testing parameters see Supporting Information. Inset: photographs of dye@matrix under $365 \mathrm{~nm}$ UV lamp and bright illustrations after removal of UV lamp.

A
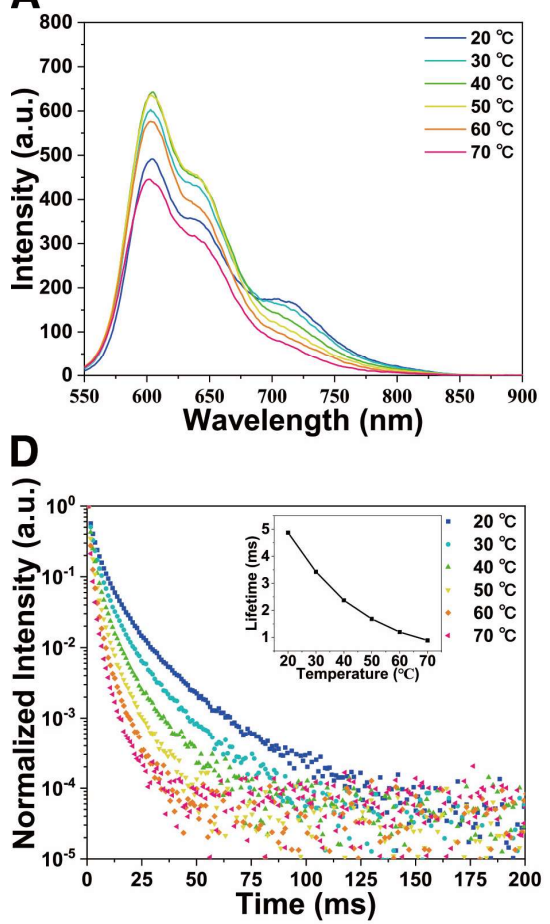

B
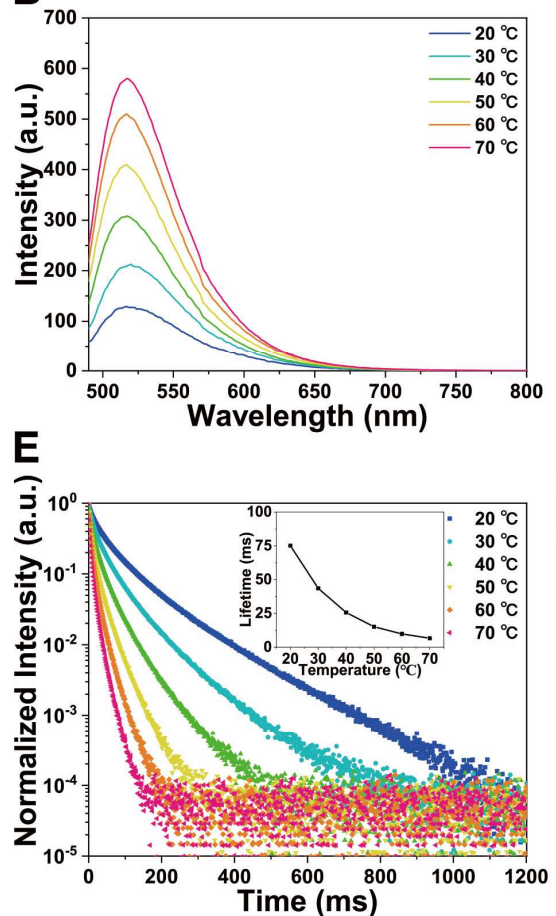
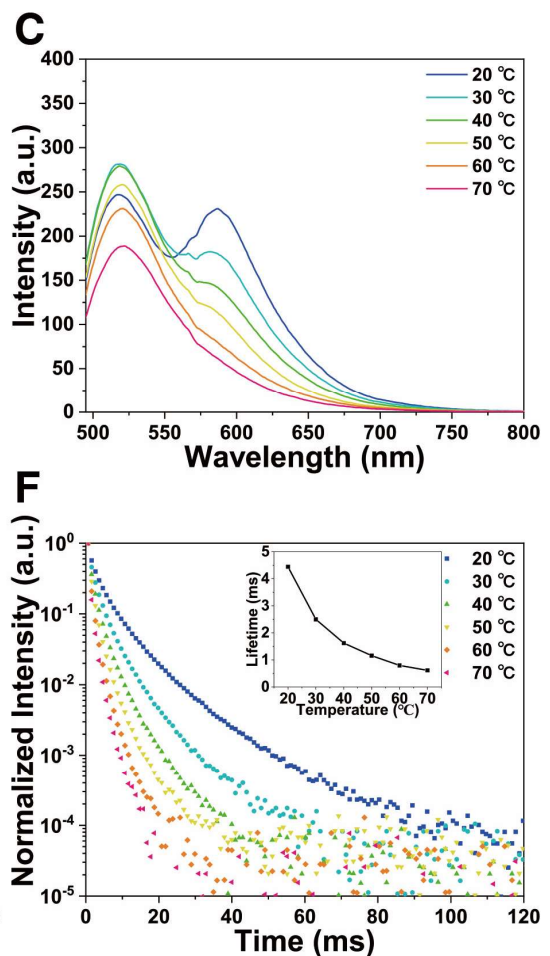

Fig. 3. Delayed spectra (smoothed) of (A) RhB@PAB; (B) PF@PAC and (C) PF@PAB at different temperatures. Lifetime measurements of (D) RhB@PAB; (E) PF@PAC and (F) PF@PAB at different temperatures. Inset: calculated lifetimes with elevating temperature

Table 1. Photophysical properties of dye@PAB materials.

\begin{tabular}{cccccc}
\hline Dye & $\lambda_{\text {ex }}(\mathrm{nm})$ & $\lambda_{\mathrm{f}}(\mathrm{nm})$ & $\lambda_{\mathrm{p}}(\mathrm{nm})$ & $\tau_{\mathrm{p}}(\mathrm{ms})$ & $\Phi_{\mathrm{PL}}(\%)$ \\
\hline NpA & 340 & 363 & 555 & 3.721 & 4.40 \\
$\mathrm{~S}-\mathrm{BN}$ & 338 & 372 & 528 & 25.918 & 17.2 \\
$\mathrm{HCM}$ & 337 & 470 & 538 & 10.657 & 31.6 \\
$\mathrm{QN}$ & 336 & 361 & 510 & 12.971 & 13.6 \\
$\mathrm{RhB}$ & 524 & 600 & 700 & 6.097 & 27.1 \\
$\mathrm{PF}$ & 484 & 509 & 581 & 4.946 & 1.80 \\
\hline
\end{tabular}




\section{Supplementary Materials for}

Activating Room-Temperature Phosphorescence of Organic Luminophores via

External Heavy-Atom Effect and Rigidity of Ionic Polymer Matrix

Zi-Ang Yan, Xiaohan Lin, Siyu Sun, Xiang Ma*, He Tian

Correspondence to: maxiang@ecust.edu.cn

General experimental procedures

Materials

Figs. S1 to S6

Table S1 


\section{Experimental Procedures}

\section{$\underline{\text { General }}$}

The UV-Vis absorption spectra were obtained on an Agilent Cary 60 spectrophotometer. Prompt spectra, delayed spectra and lifetimes were recorded on an Agilent Cary Eclipse spectrophotometer. Quantum yields were measured with HAMAMATSU Absolute PL quantum yield spectrometer C1 1347-11. X-ray powder diffraction (XRPD) was performed on a Rigaku 18KW/D/max2550VB/PC Rotating Anode X-ray Powder Diffractometer.

Photomultiplier (PMT) voltages, slits and other parameters are set to same levels when comparing between emission spectra (e.g., between dye@PAC and dye@PAB; between different temperatures). For dye@matrix (dye=NpA, S-BN, HCM, QN, RhB, PF), testing parameters are listed as follows unless otherwise stated. Prompt spectra: fluorescence mode, excitation slit $=5 \mathrm{~nm}$, emission slit $=5 \mathrm{~nm}$, PMT voltage $=550 \mathrm{~V}, 450 \mathrm{~V}, 450 \mathrm{~V}, 550 \mathrm{~V}, 500 \mathrm{~V}$ and $500 \mathrm{~V}$, respectively. Delayed spectra: phosphorescence mode, delay time $=0.1 \mathrm{~ms}$, gate time $=2.0 \mathrm{~ms}$, excitation slit $=10$ $\mathrm{nm}$, emission slit $=10 \mathrm{~nm}$, PMT voltage $=650 \mathrm{~V}, 700 \mathrm{~V}, 575 \mathrm{~V}, 550 \mathrm{~V}, 700 \mathrm{~V}$ and $625 \mathrm{~V}$, respectively (for three temperature-dependent spectra, PMT voltage $=700 \mathrm{~V}, 575 \mathrm{~V}, 500 \mathrm{~V}$, respectively).

\section{$\underline{\text { Materials }}$}

Poly-diallyldimethylammonium chloride $(\mathrm{MW}=100000)$ was purchased from Adamas-beta as 35 wt.\% aqueous solution and purified with dialysis. 1,8-naphthalimide (NpA) and 3,6diaminoacridine hydrochloride (PF) were purchased from Bidepharm and used without further purification. (S)-1,1'-bi-2-naphthol (S-BN), 6,7-dihydroxycoumarin (HCM), quinine sulfate (QN) and rhodamine $\mathrm{B}(\mathrm{RhB})$ were purchased from Adamas-beta and used without further purification. All other reagents were commercially available and used as supplied without further purification. Solvents were purified according to standard laboratory methods. 
Purification and preparation of PAB and PAC used for doping: $15 \mathrm{~mL}$ of $35 \mathrm{wt} . \%$ PAC solution mentioned above was dialyzed against ultra-pure water $(5 * 1000 \mathrm{~mL})$ for two days to give pure PAC solution. Another portion of $15 \mathrm{~mL}$ PAC solution was dialyzed against $1000 \mathrm{~mL}$ of $15 \mathrm{~g} \cdot \mathrm{L}^{-1} \mathrm{KBr}$ solution for $12 \mathrm{~h}$ and ultra-pure water $\left(3^{*} 1000 \mathrm{~mL}\right)$ for one day. This ion-exchange process was repeated three times before concentration and further dialysis against ultra-pure water $(5 * 1000 \mathrm{~mL})$ for two days to remove excess $\mathrm{KBr}$ (as is indicated with addition of $\mathrm{AgNO}_{3}$ solution into water outside the dialysis bag yielding no precipitate after the third portion of $1000 \mathrm{~mL}$ water) to give pure PAB solution. To verify the effectiveness of this ion-exchange method, the process was repeated only once (instead of three times) and with only a fifth $\mathrm{KBr}$ before removal of excess salts. The polymer solution forms yellow precipitate upon addition of $\mathrm{AgNO}_{3}$ solution, indicating that only once ion-exchange process can replace most of $\mathrm{Cl}^{-}$with $\mathrm{Br}^{-}$while we performed up to three times. The resulting $\mathrm{PAC}$ and $\mathrm{PAB}$ solutions were respectively concentrated up to dryness, and further dried in a vacuum drying oven at $40^{\circ} \mathrm{C}$ for three days to yield two transparent solids. The solids were kept in a glass dryer with $\mathrm{P}_{2} \mathrm{O}_{5}$ as desiccant and $\mathrm{NaOH}$ as acidic gas absorbent for further use.

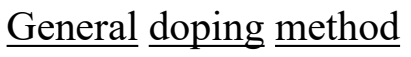

The molar amount of polymer mentioned below is expressed in the molar amount of monomer units of the corresponding polymer.

$40.0 \mu \mathrm{L}$ of $2.25 \mathrm{~mol} \cdot \mathrm{L}^{-1} \mathrm{PAB}$ aqueous solution (or $39.1 \mu \mathrm{L}$ of $2.30 \mathrm{~mol} \cdot \mathrm{L}^{-1} \mathrm{PAC}$ aqueous solution) was mixed with $20.0 \mu \mathrm{L}$ of $15.00 \mathrm{mmol} \cdot \mathrm{L}^{-1}$ (for $\mathrm{NpA}$, the concentration was $7.50 \mathrm{mmol} \cdot \mathrm{L}^{-}$ ${ }^{1}$ ) dye solution (aqueous or ethanol) to form a homogenous solution. The solution was added to a $\Phi 9$ $\mathrm{mm} * 0.5 \mathrm{~mm}$ quartz cell. Solvent needs to be evaporated slowly to avoid cracks. The cell was therefore put in an electric thermostatic drying oven at $40^{\circ} \mathrm{C}$ for $20 \mathrm{~min}$ before the temperature was set to $60^{\circ} \mathrm{C}$ for $10 \mathrm{~min}$. The resulting film was put into a vacuum drying oven at $30^{\circ} \mathrm{C}$ for two days for further drying. The dried film was kept in a glass dryer and directly used for measurement. 


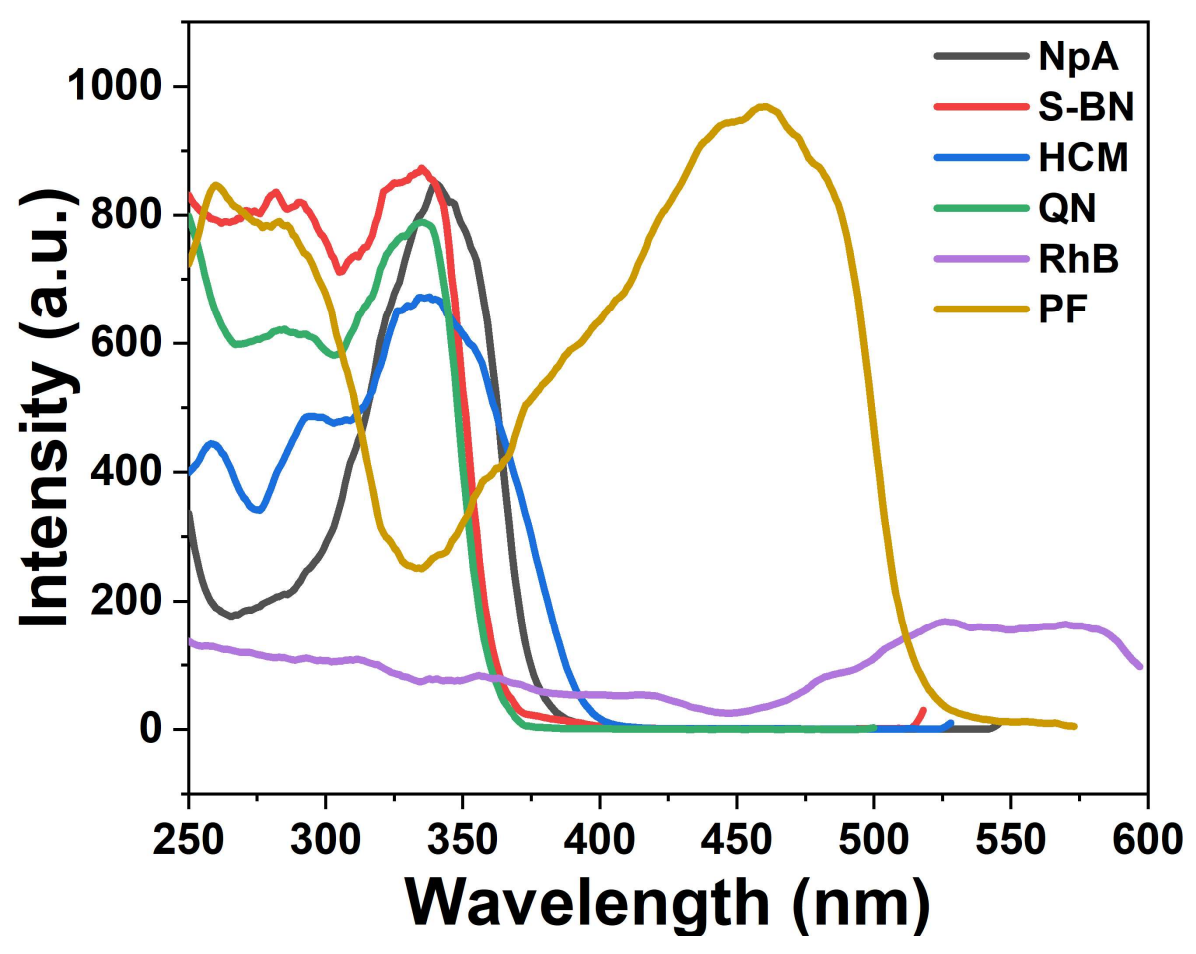

Fig. S1.

Excitation spectra (smoothed) of dye@PAB. Phosphorescence mode: delay time $=0.1 \mathrm{~ms}$, gate time $=2.0 \mathrm{~ms}$, excitation slit $=10 \mathrm{~nm}$, emission slit $=10 \mathrm{~nm}$, emission wavelength is set according to the phosphorescence emission maxima of these materials, respectively. 

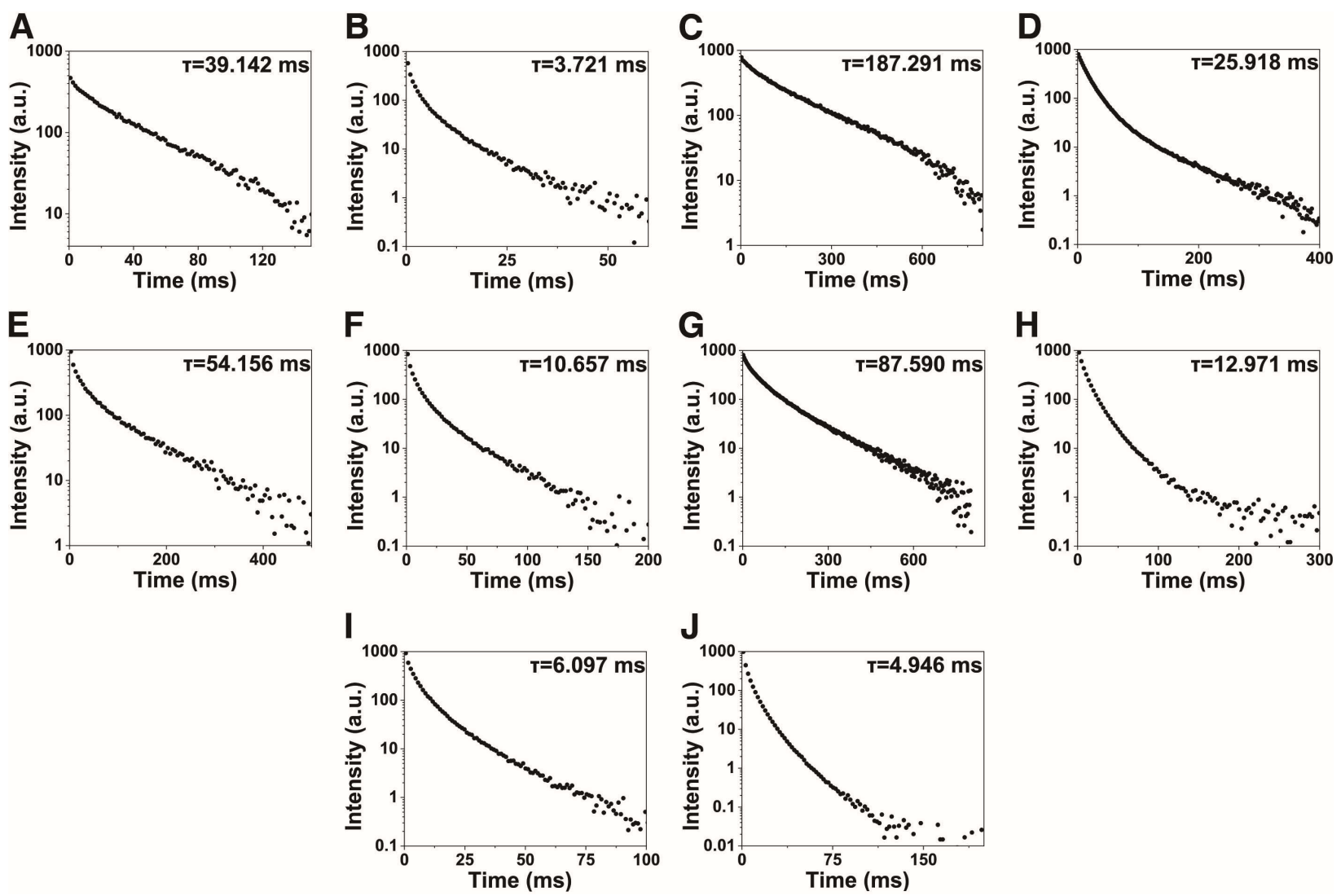

Fig. S2.

Phosphorescence lifetime measurements of (A) NpA@PAC, (B) NpA@PAB, (C) S-BN@PAC, (D) S-BN@PAB, (E)HCM@PAC, (F) HCM@PAB, (G) QN@PAC, (H) QN@PAB, (I) RhB@PAB and (J) PF@PAB. 

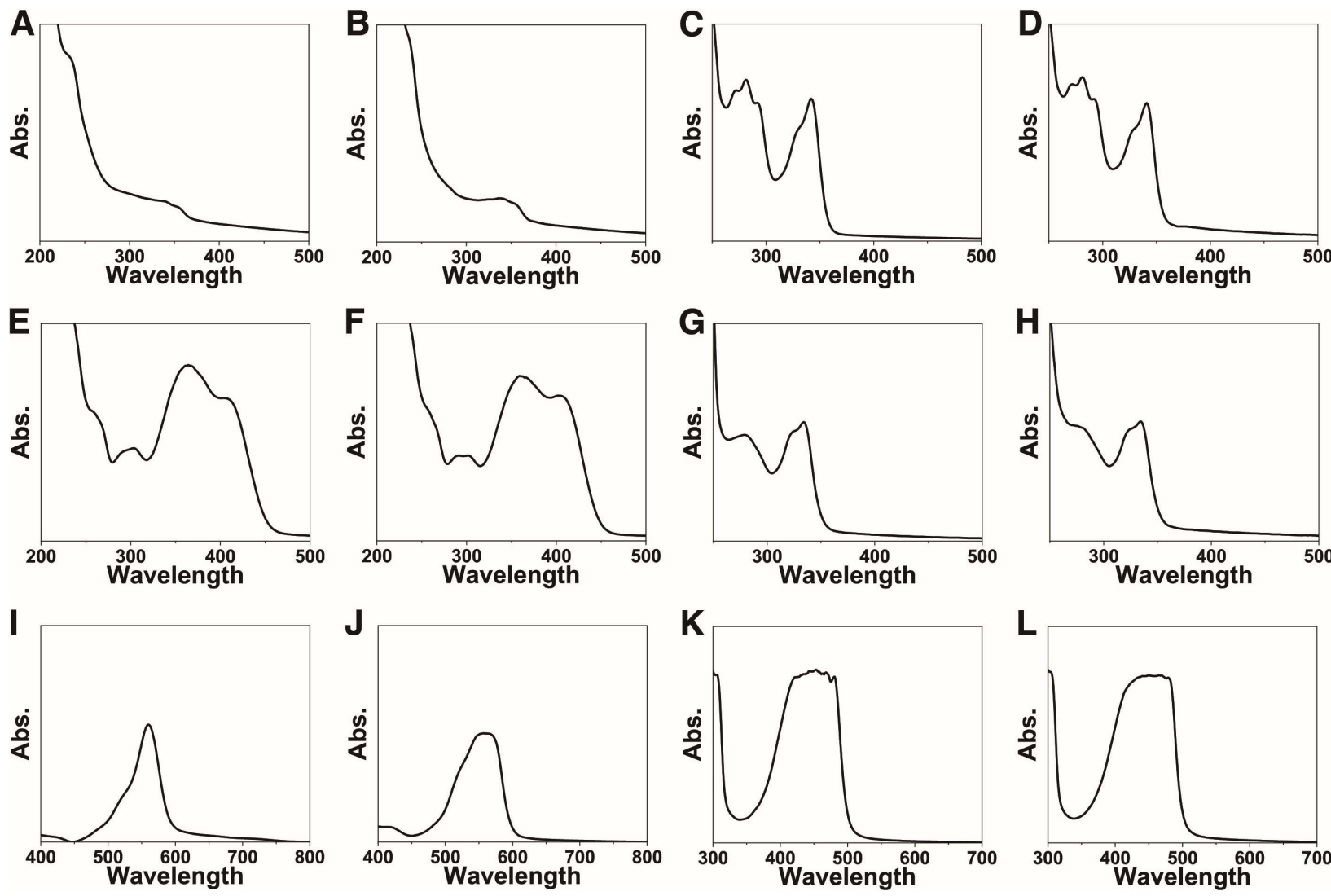

Fig. S3.

UV-Vis absorption spectra of (A) NpA@PAC, (B) NpA@PAB, (C) S-BN@PAC, (D) S-BN@PAB,

(E)HCM@PAC, (F) HCM@PAB, (G) QN@PAC, (H) QN@PAB, (I) RhB@PAC, (J) RhB@PAB, (K) PF@PAC and (L) PF@PAB. 


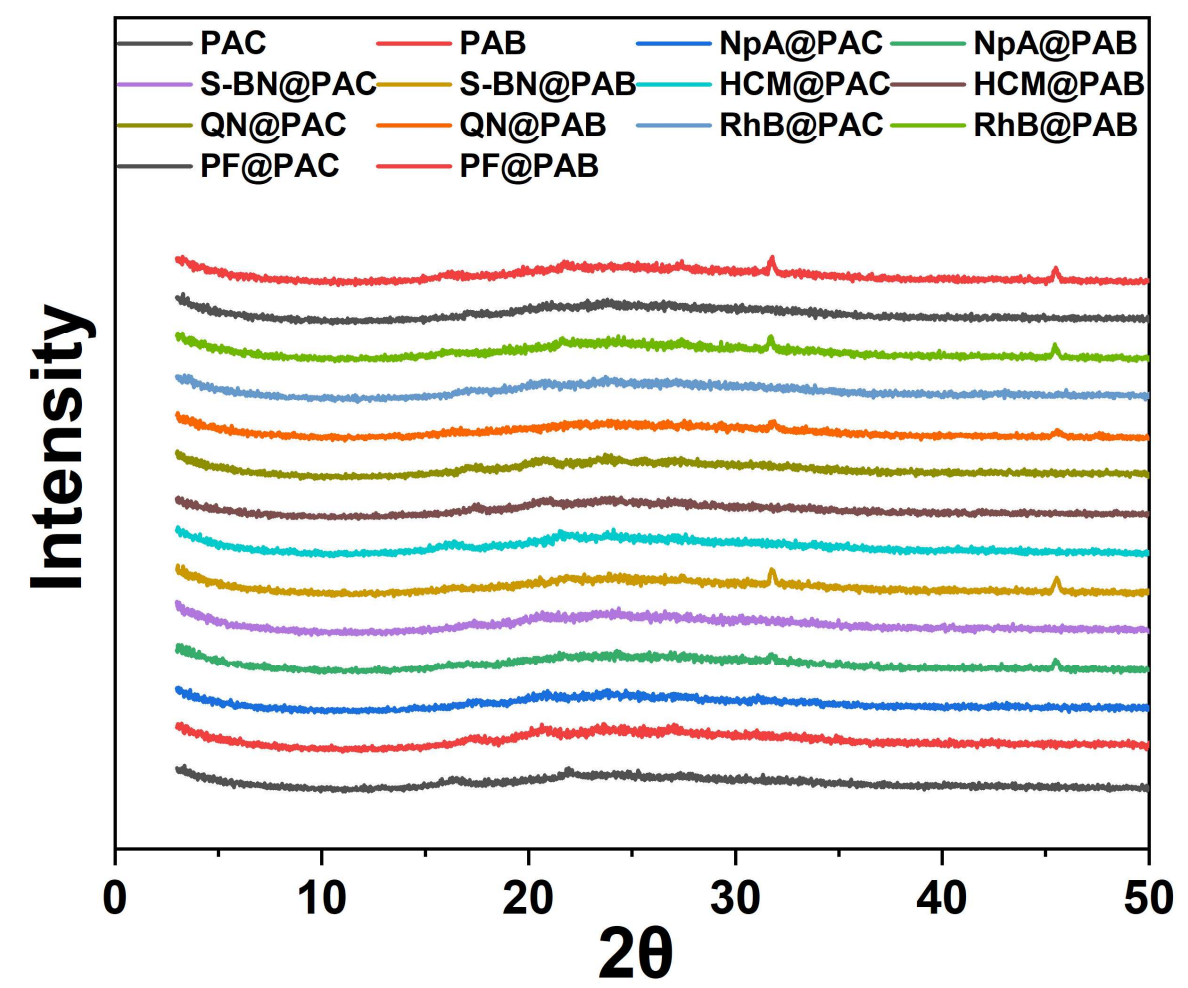

Fig. S4.

X-ray powder diffraction (XRPD) patterns of PAC, PAB and dye@matrix. 


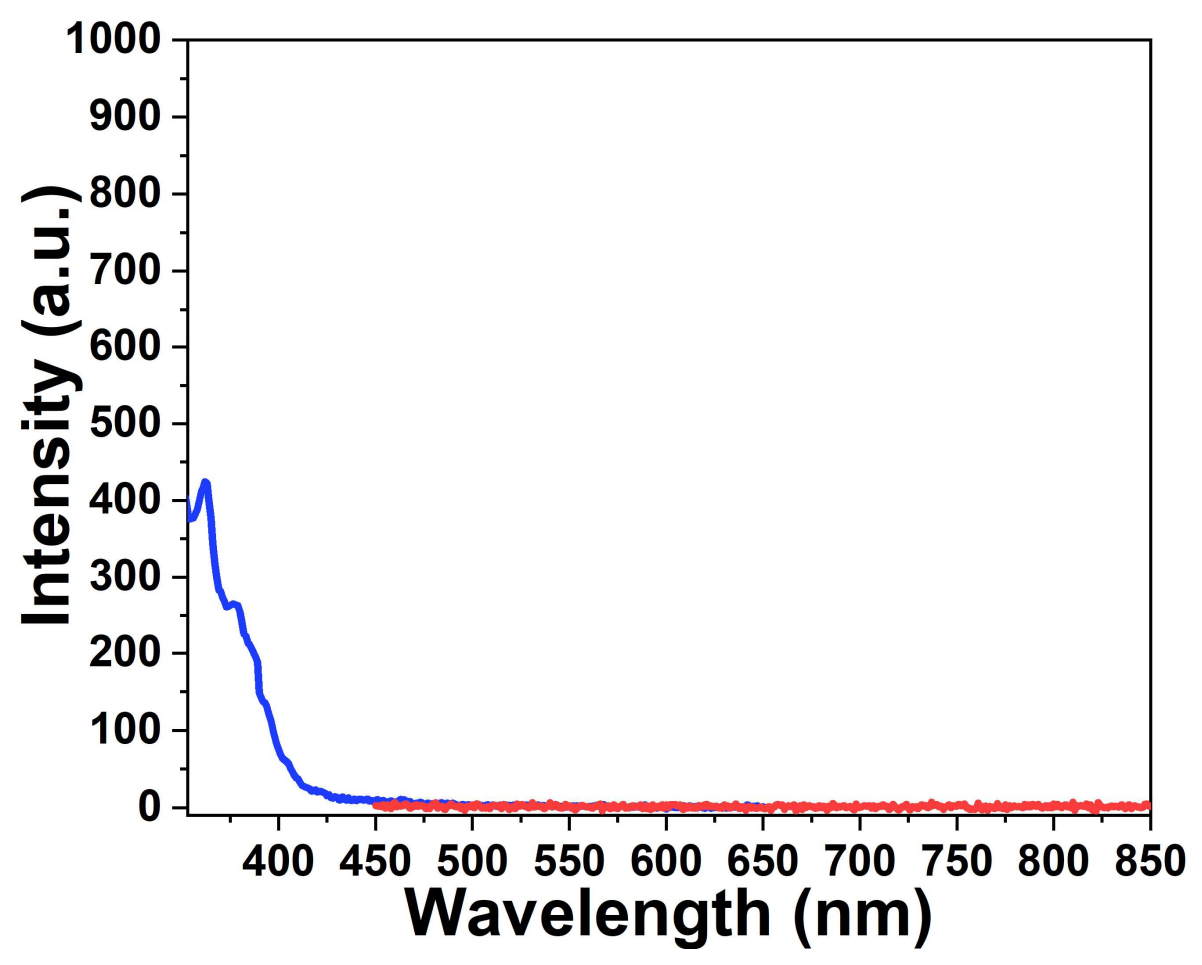

Fig. S5.

Prompt (blue line) and delayed (red line) spectra of wet NpA@PAB. 


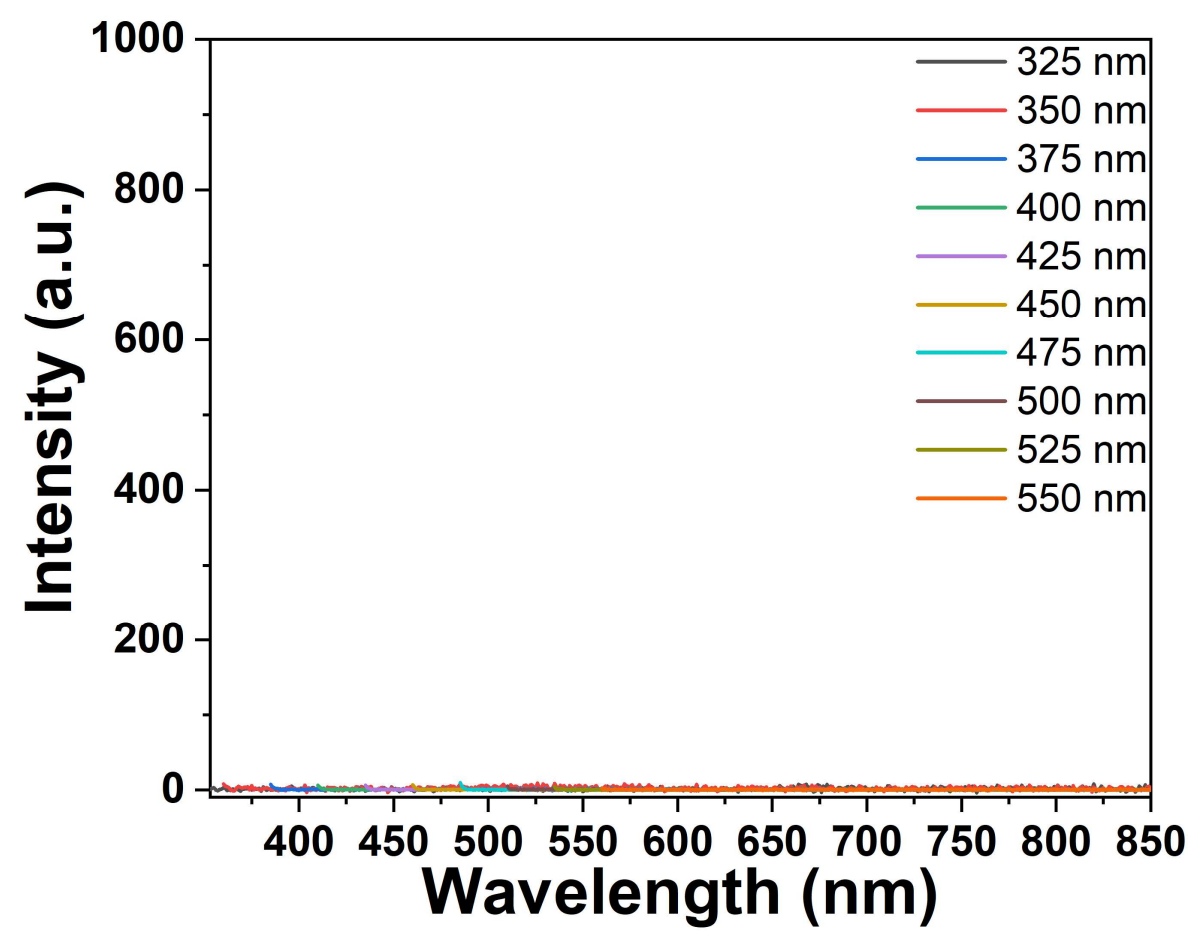

Fig. S6.

Delayed spectra of Glu@PAB at different excitation wavelengths. Photomultiplier voltage = 800 V 
Table S1.

Phosphorescence lifetimes of dye@PAC and dye@PAB, for comparison.

\begin{tabular}{ccc}
\hline Dye & $\tau_{\mathrm{p}}$ in PAC $(\mathrm{ms})$ & $\tau_{\mathrm{p}}$ in PAB $(\mathrm{ms})$ \\
\hline $\mathrm{NpA}$ & 39.142 & 3.721 \\
$\mathrm{~S}-\mathrm{BN}$ & 187.291 & 25.918 \\
$\mathrm{HCM}$ & 54.156 & 10.657 \\
$\mathrm{QN}$ & 87.590 & 12.971 \\
$\mathrm{RhB}$ & Not detected $^{[\mathrm{a}]}$ & 6.097 \\
$\mathrm{PF}$ & Not detected $^{[\mathrm{b}]}$ & 4.946
\end{tabular}

[a] Phosphorescence is too weak to be detected. [b] Phosphorescence is so weak that it is covered by delayed fluorescence. 